R. ESSALMANI, S. SOULIER, N. BESNARD, M. HUDRISIER, J . COSTA DA SILVA, J.-L. VILOTTE

INRA, Laboratoi re de Généti que Bi ochi mi que et de Cytogénéti que, 78352 J ouy-en-J osas cedex

email : vi lotte@bi otec.jouy.inra.fr

\title{
Données de base sur la transgenèse
}

\begin{abstract}
Résu mé. La transgenèse permet d'i ntrodui re dans le génome d'un ani mal un fragment d'ADN qui sera ensui te transmi s de générati on en génération. Elle uti li se différentes approches méthodologi ques. Certai nes, encore li mi tées à peu d'espèces, permettent des modi fi cati ons très fi nes du génome La transgenèse, associée au développement de la biologie moléculai re, offre des appli cati ons multi ples tant dans la recherche fondamentale qu'appli quée. Elle est de fait deve nue un outi I indi spensable pour l'analyse de la régulati on de l'expressi on des gènes et la compréhensi on de leur fonction.
\end{abstract}

Les premiers travaux publiés sur la transgenèse remontent à près de 20 ans (J aenisch 1976, Gordon et al 1980). Dès 1981, l'adjectif transgénique fut attribué à des animaux dans le génome desquels avait été introduit expérimentalement un fragment d'ADN qui pouvait être transmis à leur descendance. Très vite, cet outil est apparu extrêmement puissant pour aborder, de façon non réductrice, des études sur la fonction et la régulation de l'expression de gènes, mais également pour l'obtention d'animaux présentant des phénotypes impossibles à obtenir par sélection. La première et très médiatisée démonstration des énormes potentialités offertes par cette technique fut l'obtention de souris « géantes » exprimant le gène de l'hormone de croissance de rat (Palmiter et al 1982).

Depuis cette époque, où deux techniques pour introduire l'ADN étranger dans le génome de l'hôte étaient disponibles, la micro-injection et l'infection rétrovirale, d'autres méthodes sont progressivement apparues, dont l'utilisation des cellules embryonnaires totipotentes (Gossler et al 1986, Robertson et al 1986), permettant une manipulation de plus en plus fine du génome. De même, la transposition à différentes espèces des méthodologies souvent mises au point sur un modèle murin, a rapidement vu le jour en s'adaptant aux caractéristiques physiologiques de reproduction des animaux concernés.

Cet article aborde succinctement les différentes méthodologies de transgenèse puis, avec les outils de plus en plus perfectionnés de la biologie moléculaire, certaines potentialités offertes par cette approche, sans en décrire les applications déjà réalisées.

\section{1 / Méthodes de transfert de gènes chez la souris. Transposition aux autres espèces}

\section{1 / La micro-injection}

La micro-injection consiste à injecter dans un des pronuclei d'un œuf fécondé (habituellement le pronucleus mâle car il est le plus accessible), quelques picolitres d'une solution tamponnée renfermant plusieurs centaines de copies du fragment d'ADN que I'on désire intégrer dans le génome de l'hôte. Afin de faciliter l'intégration de cet ADN, celui-ci doit se présenter sous forme linéaire. Après avoir été micro-injectés, les œufs sont introduits dans le tractus génital de souris pseudogestantes. Une faible proportion de ces œufs (10 à $20 \%$ en moyenne) se développera pour donner naissance à des souriceaux. Le caractère transgénique de ces animaux sera révélé par la détection, dans leur ADN, du fragment micro-injecté (ou transgène). Environ 15 à $25 \%$ des animaux analysés seront porteurs du transgène, ce pourcentage pouvant varier considérablement d'une expérience à l'autre.

Cette méthode ne permet ni le contrôle du site d'intégration (qui semble aléatoire, même si cette interprétation a récemment été remise en question), ni celui du nombre de copies intégrées du transgène. On observe habituellement un seul site d'intégration par animal, à proximité duquel certains réarrangements de I'ADN de l'hôte peuvent intervenir (Rijkers et al 1994). Il été rapporté que chez la souris, le caractère aléatoire de cette intégration provoque la mutagénèse d'un gène endogène dans près de $5 \%$ des cas (Meisler 1992). Le mécanisme sous- 
jacent à l'intégration d'un transgène reste peu connu, mais semble faire appel à des phénomènes de réparation par ligation de cassures existantes dans I'ADN de I'hôte (Bishop et Smith 1989). Lorsque plusieurs copies du transgène sont intégrées dans un même site, elles sont souvent orientées en tandem. Cette disposition refléterait la formation, avant leur intégration, de " chaînes » composées, par recombinaison homologue, de copies du transgène. Malgré I'injection au stade pronuclei, le caractère mosaïque ${ }^{(1)}$ souvent observé des animaux transgéniques ainsi obtenus suggère que l'intégration de l'ADN s'effectue après la première division cellulaire (Whitelaw et al 1993). Ce phénomène se traduit (et est habituellement révélé) par un taux de transmission du transgène à la génération suivante souvent inférieur à $50 \%$.

La transposition de la micro-injection aux autres espèces de mammifères s'est effectuée avec succès dès les années 80 (Hammer et al 1985), même si certaines difficultés sont apparues comme, par exemple, l'opacité du cytoplasme de certains œufs qui necessite de les centrifuger afin de visualiser les pronuclei. Cependant, l'efficacité de cette technique s'est souvent avérée bien plus faible chez ces espèces que chez la souris. Cette observation associée au coût d'élevage, notamment des femelles receveuses d'embryons, limite, chez les gros animaux, l'application de la transgénèse par microinjection. Plusieurs méthodologies ont été employées dans le but de diminuer son coût : les œufs peuvent être obtenus à partir d'ovaires prélevés dans des abattoirs ; les ovocytes maturés et fécondés in vitro. L'identification précoce des embryons transgéniques diminuerait considérablement le nombre de femelles receveuses. Elle implique souvent le développement des œufs in vitro ou dans des oviductes de lapins jusqu'au stade morula ou blastocyste. II est alors possible de prélever quelques cellules de l'embryon pour rechercher la présence du transgène par amplification de son ADN (PCR) ou par hybridation in situ (FISH). Cependant, le résultat d'une telle sélection est souvent décevant. Deux approches alternatives semblent plus prometteuses : la co-injection d'une construction comprenant un gène rapporteur, placé sous la dépendance d'un promoteur actif à un stade pré-implantatoire seulement après son intégration dans le génome, dont l'expression peut être détectée par des méthodes non invasives (Menck et al 1998), ou l'analyse du fluide amniotique réalisée en début de gestation (Hyttinen et al 1994).

La micro-injection est la technique la plus utilisée pour l'obtention de poissons transgéniques. L'impossibilité d'accéder au noyau conduit alors à réaliser une injection cytoplasmique d'un grande quantité de copies du transgène (plusieurs milliers). Si le pourcentage d'alevins transgéniques obtenu est souvent important, pouvant atteindre $50 \%$, ces poissons sont caractérisés par leur fort mosaïcisme pour le transgène. Ce phénomène, qui résulte de la persistance de I'ADN sous forme extra-chromosomique pendant plusieurs divisions avant son intégration, peut malheureusement se traduire par la transmission du transgène à la génération suivante à des fréquences très faibles (en moyenne seulement $10 \%$ des poissons transgéniques transmettent le transgène à moins de $15 \%$ de leurs descendants). Ce mosaïcisme peut être réduit en associant à
I'ADN injecté des peptides d'adressage nucléaire (Collas et Aleström 1998). Chez les oiseaux, cette technique reste très peu utilisée. Elle implique la maîtrise de la culture ex vivo d'un œuf depuis le stade unicellulaire. D'autre part, comme dans le cas des poissons, l'opacité de l'œuf oblige à effectuer une injection cytoplasmique. Peu de laboratoires semblent à même d'utiliser cette méthodologie chez les oiseaux, mais la transmission germinale d'un transgène a pu être obtenue par cette voie (Love et al 1994) avec des rendements très faibles comparativement à ce qui est observé chez la souris.

\section{2 / L'infection rétrovirale}

La possibilité d'incorporer de l'ADN d'origine rétrovirale dans le génome de la souris à un stade précoce du développement embryonnaire afin qu'ils colonisent la lignée germinale a été démontrée dès 1976 (Jaenisch 1976). L'utilisation de tels vecteurs pose le problème de leur innocuité et, pratiquement, les vecteurs rétroviraux ont été les plus fréquemment utilisés jusqu'ici et le développement de vecteurs adénoviraux pourrait être envisageable. Leur innocuité est assurée par la délétion des gènes permettant au virus d'assurer sa propre réplication et leur remplacement par le gène d'intérêt. Ces éléments manquants sont fournis en trans lors de la construction et la multiplication des vecteurs par l'intermédiaire de cellules dites helper qui synthétisent ces protéines. II n'en demeure pas moins que la taille des gènes qui peuvent être incorporés dans ces vecteurs est limitée pour permettre l'encapsidation du génome.

L'incorporation de l'ADN d'un rétrovirus dans le génome de l'hôte s'effectue au cours d'une division cellulaire, dans un site qui semble également aléatoire, même s'il est souvent localisé à proximité de régions sensibles à la DNAse (Rijkers et al 1994). Cela se traduit par l'obtention d'animaux transgéniques à des taux équivalents à ceux obtenus par micro-injection, mais qui sont très mosaïques. Chez ces animaux, plusieurs sites d'intégration peuvent être détectés au sein d'une même cellule, entre cellules d'un même tissu ou de deux tissus distincts, chaque site renfermant une seule copie de I'ADN rétroviral. L'infection rétrovirale a été relativement peu utilisée en transgénèse chez les mammifères, présentant moins d'intérêt que la micro-injection. Cependant, des résultats récents utilisant l'infection non plus sur l'embryon mais sur des ovocytes, ont montré l'obtention d'un fort pourcentage de veaux transgéniques peu ou pas mosaïques (Chan et al 1998). A l'inverse des rétrovirus, les adénovirus peuvent infecter des cellules qui ne se divisent pas. Ils ont été utilisés avec succès pour infecter des œufs au stade pronuclei et les souris transgéniques résultantes présentaient un site unique d'intégration, peu ou pas de mosaïcisme (Tsukui et al 1996). Ils s'intègrent cependant à faible fréquence dans le génome de l'hôte.

Ces deux expériences très prometteuses, qui demandent à être répétées, pourraient en relancer l'intérêt.

L'infection rétrovirale est la principale méthode utilisée pour obtenir des oiseaux transgéniques. L'infection de l'embryon est alors réalisée dans l'œuf pondu. Les oiseaux transgéniques obtenus 
sont fortement mosaïques, mais il est néanmoins possible d'en dériver des lignées stables. Chez les poissons, si l'emploi de rétrovirus permet l'obtention de forts taux de transgéniques, leur utilisation reste limitée à des études de mutagénèse d'insertion et nécessite l'usage de virus à spectre très large.

\section{3 / L'utilisation de cellules embryonnaires totipotentes}

Les cellules embryonnaires totipotentes, ou celIules ES (pour : embryonic stem), sont des cellules issues du bouton embryonnaire d'un blastocyste ou d'un embryon au stade morula, qui peuvent être maintenues en culture sans se différencier ( $E$ vans et Kaufman 1981, Martin 1981). Ces cellules, une fois réintroduites dans des embryons, participent à l'élaboration d'un animal chimérique. Leur totipotence leur permet en théorie de coloniser tous les tissus du nouvel animal, y compris la lignée germinale. Au cours de leur phase de culture in vitro, ces cellules peuvent être manipulées par des techniques classiques de transfection afin d'introduire I'ADN étranger. II est possible alors de sélectionner les clones transformés, sans altération de la totipotence des cellules. Ainsi, il est envisageable de rechercher des phénomènes rares d'intégration, telle une intégration ciblée par recombinaison homologue. L'utilisation de ces cellules permet donc une manipulation fine du génome, pouvant aller de l'invalidation d'un gène à la simple substitution d'une base par une autre. Les cellules ES ont également été utilisées pour incorporer, dans des souris, des chromosomes humains entiers par fusion avec des microcellules. La transmission germinale du chromosome 2 humain par des souris transgéniques a ainsi été obtenue (Tomizuka et al 1997).

Cependant, cette méthodologie reste délicate d'emploi. Les cellules ES restent peu définies et, malgré leur relative stabilité, ont tendance à se différencier. Le chimérisme ${ }^{(2)}$ des animaux obtenus se traduit par un très faible taux de transmission du caractère sélectionné in vi tro à la génération suivante. La production, à partir de cellules ES, d'embryons uniquement constitués par elles a récemment été décrite suite à l'introduction de ces cellules dans des blastocystes tétraploïdes (Wang et al 1997).

L'isolement de cellules ressemblant aux cellules ES a été obtenu dans de nombreuses espèces : hamster, rat, porc, lapin, poulet, truite, vison, vache, médaka et plusieurs primates dont I'Homme. Ces cellules ont la particularité d'être pluripotentes et, dans certains cas, ont donné naissance à des animaux chimères. Cependant, aucune n'a à ce jour été décrite comme pouvant coloniser la lignée germinale.
Alternativement aux cellules ES, des recherches ont porté sur l'utilisation de cellules primordiales germinales, isolées à partir de gonades fœtales ou de sang d'embryons. Le caractère totipotent de telles cellules a pu être démontré dans certaines espèces, mais leur faculté à pouvoir être maintenues en culture, à être transformées et sélectionnées sans perdre cette totipotence, n'est pas clairement établie.

\section{4 / Le clonage}

Le clonage consiste soit à transférer le noyau d'une cellule dans un ovocyte énucléé, soit à fusionner ces deux cellules. II est alors possible d'obtenir la formation d'un embryon qui se développe normalement. Cette opération peut être répétée a partir de plusieurs cellules d'un même animal. La possibilité ainsi offerte d'utiliser des noyaux de cellules différenciées qui sont ainsi déprogrammés, ouvre de nouvelles perspectives zootechniques, de nouvelles applications thérapeutiques et revolutionne notre vision de la différenciation des cellules animales. Après les succès obtenus par l'équipe d'E dimbourg à partir de la fusion de cellules embryonnaires fœtales (Campbell et al 1996), puis à partir de cellules adultes avec la naissance de la très médiatisée Dolly (Wilmut et al 1997), la naissance de Polly est presque passée inaperçue (Schnieke et al 1997). Polly représente pourtant la première brebis transgénique obtenue par transfection de cellules fœtales fibroblastiques, sélection et analyse des clones obtenus, puis clonage. Chez les ruminants, le clonage ouvre une nouvelle voie de transgénèse qui concurrence déjà fortement la micro-injection et pourrait suppléer dans ces espèces l'absence de cellules ES. Par rapport à la micro-injection, d'une part elle serait financièrement moins coûteuse, car utilisant moins d'animaux, d'autre part elle éviterait le possible mosaïcisme des fondateurs. Son désavantage pour la transgénèse additive est la nécessité de sélectionner les clones transformés, donc d'introduire un marqueur de sélection en même temps que le transgène. Des méthodes théoriques devraient permettre dans un deuxième temps d'éliminer ce gène marqueur, mais leur application n'a pas encore été réalisée. De nombreuses expériences sont en cours pour essayer, à partir de cellules embryonnaires fœtales en culture, d'obtenir des intégrations ciblées de transgènes par recombinaison homologue. Des résultats, obtenus par la firme PPL (Edimbourg, Ecosse), font état du succès de cette approche dans l'espèce ovine. Ces cellules pourraient donc remplacer avantageusement de potentielles cellules ES chez les rumi-nants. E n effet, la technique du clonage par fusion cellulaire a également été utilisée avec succès chez la vache (Kato et al 1998) et la chèvre (Baguisi et al 1999).

Les quatre principales techniques de trangenèse.

\begin{tabular}{|c|c|c|c|c|c|}
\hline Technique & Site d'intégration & Nombre de copies & Mammifères & Poissons & Oiseaux \\
\hline Micro-injection & Aléatoire & Aléatoire & Possible & Possible & Difficile \\
\hline Infection virale & Aléatoire (?) & Une & Peu utilisée & Peu utilisée & Possible \\
\hline Cellule ES & $\begin{array}{l}\text { Ciblage possible } \\
\text { Chimérisme }\end{array}$ & Aléatoire/Unique & Souris & Non disponible & Pas validée \\
\hline Clonage & Ciblage possible & Aléatoire/Unique & Validée & Pas validée & Non disponible \\
\hline
\end{tabular}

(2) Animal transgénique chimérique : animal transgénique comportant des cellules provenant d'au moins deux animaux distincts. 
Paradoxalement, cette technique semble plus avancée chez les ruminants que chez la souris. Les difficultés rencontrées dans cette espèce pourrait être liées à un développement plus rapide de l'embryon murin dont l'activation du génome débute dès le stade deux cellules. On notera cependant le succès récent obtenu par introduction de noyaux de cellules somatiques dans des ovocytes énucléés de souris (Wakayama et al 1998).

\section{5 / Autres approches}

L'obtention d'animaux transgéniques par utilisation de spermatozoïdes préalablement mis en contact avec de l'ADN reste très discutée. L'association de I'ADN avec le spermatozoïde et sa pénétration à l'intérieur de ce dernier ont été clairement mises en évidence. Cependant, les quelques animaux transgéniques apparemment obtenus à partir de cette technique, présentaient souvent des transgènes profondément remaniés et/ou l'absence de colonisation de leur lignée germinale. Cependant, des études récentes sur l'injection de la tête de spermatozoïdes, dont la membrane a été ou non altérée, mélangée à de l'ADN dans le cytoplasme d'ovocytes de souris en métaphase II, ont permis l'obtention d'animaux transgéniques qui expriment le transgène et le transmettent par leur lignée germinale à des taux similaires à ceux observés en micro-injection (Perry et al 1999). Cette approche alternative demande cependant à être confirmée et transposée à d'autres espèces.

Récemment, l'équipe américaine de Brinster a démontré la possibilité de maintenir en culture des spermatogonies de souris avant de les réimplanter dans le testicule d'un mâle receveur - dont les propres spermatogonies ont été préalablement détruites - où ils achèvent leur maturation. II a même été possible d'effectuer la maturation de spermatogonies de rat dans un testicule de souris. $S^{\prime}$ il devient possible de maintenir ces cellules en culture assez longtemps pour permettre leur manipulation par transfection, elles constitueraient un nouveau vecteur de transgénèse. II pourrait alors être envisagé de modifier des spermatogonies issus de gros animaux puis d'en assurer la maturation dans des testicules de souris (Clouthier et al 1996).

L'utilisation des ovocytes comme cellule cible pour la transgénèse a été envisagée dans plusieurs espèces, mais apparemment sans grand succès.

\section{2 / Potentialités de la transgenèse}

La transgenèse s'est imposée comme un outil essentiel pour l'étude de la régulation et de la fonction des gènes. Elle permet en effet d'effectuer ces analyses dans la complexité d'un organisme complet, à des stades divers du développement, et est de fait beaucoup plus informative que les seules expériences menées en culture de cellules. Outre des recherches fondamentales comme la possibilité d'isoler des gènes exprimés au cours du développement ou impliqués dans le contrôle d'une fonction biologique particulière par la technique de « piégeage de gènes », la transgenèse offre également des possibilités de recherche plus appliquée (création d'animaux modèles pour des études biomédicales, production de protéines recombinantes, de cellules ou d'organes pour des xénogreffes) et plus zootechnique : résistance à certaines maladies, modification de la composition du lait ou amélioration de fonctions biologiques d'intérêt agronomique. Dans ce dernier exemple, l'apport de la transgenèse restera sans doute plus limité : d'abord les caractères étudiés sont souvent quantitatifs et polygéniques et la transgénèse ne peut véritablement avoir un impact intéressant que par l'utilisation de gènes à effet majeur, ensuite son intérêt par rapport à une sélection génétique classique devra être clairement établi, enfin, l'acceptabilité par le public de ces animaux ou des produits qui en sont issus est un préalable indispensable.

\section{1 / Le franchissement de la barrière d'espèce et de la barrière tissulaire}

La transgenèse permet l'expression d'un gène d'une espèce dans une autre. Elle offre également la possibilité de modifier le profil d'expression du gène en question par l'utilisation de séquences régulatrices dérivées d'un autre gène. Cependant, ces applications se heurtent à la faible connaissance actuelle de la régulation de l'expression des gènes. Rares en effet sont les promoteurs pour lesquels les connaissances acquises sont telles que l'on puisse prévoir avec exactitude leur profil d'expression en transgenèse. Pour la plupart, on observe une influence de l'environnement chromatinien sur leur régulation, un phénomène appelé " effet de position ». Cet effet traduit probablement l'absence, dans les constructions utilisées, d'éléments cis-régulateur capables d'isoler le transgène.

L'intégration ciblée du transgène par recombinaison homologue permet de s'affranchir de cet effet, mais, outre que cette technique est très lourde, elle reste limitée puisque basée sur l'utilisation de cellules ES ou de la technique du clonage. Une approche alternative empirique consiste à encadrer le transgène par de longues séquences flanquantes, de plusieurs dizaines de kilobases. Cela peut être obtenu à l'aide de vecteurs de clonage spéciaux, tels des YACs, BACs, PACs ou MACs. On observe alors habituellement une expression du transgène indépendante de son site d'intégration (Schedl et al 1993). De plus, il est également possible d'utiliser ces vecteurs pour introduire ex vivo des mutations dans les gènes étudiés afin d'analyser par la suite leur incidence en transgenèse (Yang et al 1997).

\section{2 / Variations quantitatives de l'expression d'un gène}

Augmentati on del'expressi on. L'augmentation du niveau d'expression d'un gène peut être obtenue en transgenèse par micro-injection, avec cependant les restrictions mentionnées ci-dessus. Une variation quantitative transitoire du niveau d'expression d'un gène chez un même animal peut être obtenue par l'utilisation de promoteurs inductibles comme, par exemple, les promoteurs dont l'activité est induite en présence de tétracycline (Gossen et al 1994).

Diminution de l'expression. L'invalidation d'un gène peut être obtenue par recombinaison homologue dans des cellules ES ou dans des cellules fœtales de ruminants. II est également possible de limiter cette invalidation à un tissu donné. On peut, par exemple, introduire par recombinaison homologue de part et d'autre du gène deux courtes séquences de type LoxP. II suffit ensuite de cibler l'expression d'une recombinase, l'enzyme Cre, dans 
le tissu cible par transgénèse classique. Cette enzyme va alors exciser la région du génome comprise entre les deux sites LoxP, provoquant I'invalidation du gène (Selbert et al 1998).

Alternativement, une diminution du niveau d'expression d'un gène est possible par ciblage d'ARNs antisens ou de ribozymes contre ses messagers (Welch et al 1998). Malheureusement, I'amplitude de la réponse obtenue est aléatoire et non contrôlée. Il est également possible, à partir de souris porteuses d'un gène invalidé, de maîtriser son niveau d'expression en micro-injectant dans ces animaux ce gène sous dépendance d'un promoteur inductible.

L'avancement des connaissances sur la régulation de l'expression des gènes devrait permettre d'identifier des éléments cis-régulateurs impliqués dans l'amplitude de la transcription. De tels éléments pourraient alors être soit ajoutés à des gènes dont on souhaiterait augmenter l'expression, soit au contraire éliminés. Il en va de même pour des éléments qui stabilisent les ARNm et/ou augmentent l'efficacité de leur traduction.

\section{3 / Variations qualitatives}

La synthèse d'une protéine présentant certaines mutations structurales peut être obtenue en transgenèse après modification in vitro, par mutagénèse dirigée, soit de son gène, soit de son ADNC. Plusieurs possibilités se présentent alors, suivant le déterminisme génétique du caractère étudié.

Si le caractère étudié est un caractère dominant, l'expression de la protéine recombinante, en présence de la protéine homologue endogène, se fera par des techniques classiques de transgenèse, notamment par micro-injection.

Si le caractère étudié est récessif, deux possibilités existent. D'une part, même si cette technique ne semble être maîtrisée que par très peu d'équipes, il est possible d'effectuer dans des cellules ES des modifications fines de la structure d'un gène, comme, par exemple, la substitution d'un codon par un autre (Stacey et al 1994). D'autre part, il peut être envisagé d'injecter le gène muté dans des œufs de souris dont le gène endogène aura été préalablement invalidé.

\section{4 / Remaniements chromosomiques}

La transgenèse, en s'appuyant sur les techniques de recombinaison par l'intermédiaire de séquences cibles, comme par exemple le système Cre/LoxP déjà cité, permet également d'induire de profondes altérations dans la structure du génome.

Inversi on de gènes. L'ordre des gènes au sein d'un locus peut être inversé en plaçant, par recombinaison homologue, deux séquences LoxP, en orientation inverse, de part et d'autre de la région concernée. L'expression transitoire de l'enzyme Cre conduit alors à l'inversion du fragment d'ADN compris entre ces deux sites (Tanimoto et al 1999).

Déléti ons/dupli cati ons i ntrachromosomi ques. La délétion et la duplication d'une région d'un chromosome ont été réalisées après insertion, par recombinaison homologue, à une extrémité de la zone d'intérêt d'un site LoxP sur un des deux chromosomes homologues, et d'un second site LoxP, dans la même orientation, à l'autre extrémité de cette zone, sur le second chromosome. L'expression de l'enzyme Cre au cours de la prophase de la méiose conduit à une recombinaison entre chromosomes appariés par l'intermédiaire des sites LoxP. II en résulte une duplication de la région ciblée sur un des chromosomes et sa délétion dans l'autre (Hérault et al 1998).

Perte d'un chromosome La présence de deux sites LoxP, en orientation inverse, sur les deux chromosomes homologues, provoque en présence de l'enzyme Cre des recombinaisons entre chromatides sœurs. Si ces recombinaisons sont inégales, I'un des chromosomes ne présentera plus de centromère et sera donc perdu au cours de la division cellulaire tandis que l'autre, qui possédera deux centromères, sera soit également perdu au cours de la division, soit uniquement transmis à une des deux cellules filles (Lewandoski et Martin 1997).

\section{Conclusion}

L'association des techniques de transgenèse et de biologie moléculaire permet des manipulations de plus en plus fines du génome. Cependant, certaines utilisations restent limitées à quelques espèces, la souris restant dans ce domaine l'animal modèle par excellence. Le développement des techniques de clonage chez certains mammifères, notamment les ruminants, permettra sans doute de combler rapidement ce retard. Pour d'autres espèces, la physiologie de leur reproduction reste un obstacle important. Les applications actuelles de la transgenèse concernent essentiellement des études très fondamentales sur la fonction et la régulation de l'expression des gènes, ou plus appliquées touchant au domaine médical et paramédical et à la santé animale. On notera cependant l'importance potentielle de la transgénèse pour l'identification de gènes à effet majeur sur des caractères d'intérêt agronomique, comme cela a été parfaitement illustré par l'exemple de la myostatine. Le développement de la transgenèse sur des animaux d'élevage souffre encore d'un important déficit d'image.

\section{Références}

Baguisi A., Behboodi E., Melican D.T. et al,1999. Production of goats by somatic cell nuclear transfer. Nature Biotechnology, 17, 456-461.

Bishop J.O., Smith P., 1989. Mechanism of chromosomal integration of microinjected DNA. Molecular and Biological Medecine, 6, 283-298.

Campbell K.H.S., McWhir J ., Ritchie W.A., Wilmut I., 1996. Sheep cloned by nuclear transfer from a cultured cell line. Nature, 380, 64-67.
Chan A.W.S., Homan E.J., Ballou L.U., Burns J.C., Bremel R.D., 1998. Transgenic cattle produced by reverse-transcribed gene transfer in oocytes. Proceedings of the National Academy of Sciences, USA, 95, 14028-14033.

Clouthier D.E., Avarbock M.R., Maika S.D., Hammer R.E., Brinster R.L., 1996. Rat spermatogenesis in mouse testis. Nature, 381, 418-420. 
Collas P., Aleström P., 1998. Nuclear localization signals enhance germline transmission of a transgene in zebrafish. Transgenic Research, 7, 303-309.

Evans M.J ., Kaufman M.H., 1981. Establishment in culture of pluripotential cells from mouse embryos. Nature, 292, 154-156.

Gordon J.W., Scangos G.A., Plotkin D.J., Barbosa J.A., Ruddle F.H., 1980. Genetic transformation of mouse embryos by microinjection of purified DNA. Proceedings of the National Academy of Sciences, USA, 77, 7380-7384.

Gossen M., Bonin A.L., Freundlieb S., Bujard H., 1994. Inducible gene expression systems for higher eukaryotic cells. Current Opinion in Biotechnology, 5, 516-520.

Gossler A., Doetschman T., Korn R., Serfling E., Kemler R., 1986. Transgenesis by means of blastocyst-derived embryonic stem cell lines. Developmental Biology, 83, 9065-9069.

Hammer R.E., Pursel V.G., Rexroad J.R., Wall R.J., Bolt D.J., Ebert K.M., Palmiter R.D., Brinster R.L., 1985. Production of transgenic rabbits, sheep and pigs by microinjection. Nature, 315, 680-683.

Hérault Y., Rassoulzadegan M., Cuzin F., Duboule D., 1998. Engineering chromosomes in mice through targeted meiotic recombination (TAMERE). Nature Genetics, 20, 381-384.

Hyttinen J.M., Peura T., Tolvanen M., Aalto J., Alhonen L., Sinervirta R., Hamelkytö M., Myöhänen S., Jänne J., 1994. Generation of transgenic dairy cattle from transgene-analyzed and sexed embryos produced in vitro. Bio/Technology, 12, 606-608.

J aenisch R., 1976. Germ line integration and Mendelian transmission of the exogenous Moloney leukemia virus. Proceedings of the National Academy of Sciences, USA, 73, 1260-1264.

Kato Y., Tani T., Sotomaru Y., Kurogawa K., Kato J .Y., Doguchi H., Yasue H., Tsunoda Y., 1998. Eight calves cloned from somatic cells of a single adult. Science, 282, 2095-2098.

Lewandoski M., Martin G.R., 1997. Cre-mediated chromosome loss in mice. Nature Genetics, 17, 223-225.

Love J ., Gribbin C., Mather C., Sang H., 1994. Transgenic birds by DNA microinjection. Bio/Technology, 12, 60-63.

Martin G., 1981. Isolation of a pluripotent cell line from early mouse embryos cultured in medium conditioned by teratocarcinoma stem cells. Proceedings of the National Academy of Science, USA, 78, 7634-7638.

Meisler M.H., 1992. Insertional mutation of classical and novel genes in transgenic mice. Trends in Genetics, 8, 341-348.

Menck M.C., Mercier Y., Campion E., Lobo R.B., Heyman Y., Renard J .P., Thompson E.M., 1998. Prediction of transgene integration by noninvasive bioluminescent screening of microinjected bovine embryos. Transgenic Research, 7, 331-341.

Palmiter R.D., Brinster R.L., Hammer R.E., Trumbauer M.E., Rosenfeld M.G., Birnberg N.C., Evans R.M., 1982. Dramatic growth of mice that develop from eggs microinjected with metallothionein-growth hormone fusion genes. Nature, 300, 611-615.

Perry A.C.F., Wakayama T., Kishikawa H., Kasai T., Okabe M., Toyoda Y., Yanagimachi R., 1999. Mammalian transgenesis by intracytoplasmic sperm injection. Science, 284, 1180-1183.
Rijkers T., Peetz A., Rüther U., 1994. Insertional mutagenesis in transgenic mice. Transgenic Research, 3, 23-215.

Robertson E., Bradley A., Kuehn M., Evans M., 1986. Germ-line transmission of genes introduced into cultured pluripotential cells by retroviral vector. Nature, 323, 445-448

Schedl A., Montoliu L., Kelsey G., Schütz G., 1993. A yeast artificial chromosome covering the tyrosinase gene confers copy number-dependent expression in transgenic mice. Nature, 362, 258-261.

Schnieke A.E., Kind A.J., Ritchie W.A., Mycok K., Scott A.R., Ritchie M., Wilmut I., Colman A., Campbell K.H.S., 1997. Human factor IX transgenic sheep by transfer of nuclei from transfected fetal fibroblasts. Science, 278, 2130-2133.

Selbert S., Bentley D.J ., Melton, D.W., Rannie D., Lourenço P., Watson C.J., Clarke A.R., 1998. Efficient BLG-Cre mediated gene deletion in the mammary gland. Transgenic Research, 7, 387-396.

Stacey A., Schnieke A., McWhir J ., Cooper J ., Colman A., Melton D.W., 1994. Use of double gene targeting to replace the murine alpha-lactalbumin gene with its human counterpart in embryonic stem cells and mice. Molecular and Cellular Biology, 14, 1009 1016

Tanimoto K., Liu Q., Bungert J ., Engel J.D., 1999. Effects of altered gene order or orientation of the locus control region on human beta-globin gene expression in mice. Nature, 398, 344-348.

Tomizuka K., Yoshida H., Uejima H., Kugoh H., Sato K., Ohguma A., Hayasaka M., Hanaoka K., Oshimura M., Ishida I., 1997. Functional expression and germline transmission of a human chromosome fragment in chimaeric mice. Nature Genetics, 16, 133-143.

Tsukui T., Kanegae Y., Saito I., Toyoda Y., 1996. Transgenesis by adenovirus-mediated gene transfer into mouse zona-free eggs. Nature Biotechnology, 14, 982-985

Wang Z.Q. Kiefer F., Urbanek P., Wagner E.F., 1997. Generation and completely embryonic stem cell-derived mutant mice using tetraploid blastocyst injection. Mechanisms Development, 62, 137-145.

Wakayama T., Perry A.C.F., Zuccotti M., Johnson K.R. Yanagimachi R., 1998. Full-term development of mice from enucleated oocytes injected with cumulus cell nuclei. Nature, 394, 369-371.

Welch P.J ., Barber J.R., Wong-Staal F., 1998. Expression of ribozymes in gene transfer systems to modulate target RNA levels. Current Opinion in Biotechnology, 9, 486-496.

Whitelaw C.B.A., Springbett A.J ., Webster J., Clark J ., 1993. The majority of Go transgenic mice are derived from mosaic embryos. Transgenic Research, 2, 29-32.

Wilmut I., Schnieke A.E., McWhir J., Kind A.J ., Campbell K.H.S. 1997. Viable offspring derived from fetal and adult mammalian cells. Nature, 385, 810-813.

Yang X.W., Model P., Heintz N., 1997. Homologous recombination based modification in Esherichia Coli and germline transmission in transgenic mice of a bacterial artificial chromosome. Nature Biotechnology, 15, 859-865. 